\title{
Diseño y Construcción de un Inversor Trifásico acoplado a un Sistema de Transferencia Automático con Monitoreo SCADA
}

\author{
Oscar Angel Revelo Ger \\ Carrera de Electricidad \\ Universidad Técnica del Norte \\ oarevelog@utn.edu.ec \\ Anthony Raúl Revelo Clerque \\ Carrera de Electricidad \\ Universidad Técnica del Norte \\ arreveloc@utn.edu.ec \\ Jhonny Javier Barzola Iza \\ Carrera de Electricidad \\ Universidad Técnica del Norte \\ jjbarzola@utn.edu.ec
}

Con el avance de la tecnología en muchas empresas de producción actuales han optado por el uso de energías que son amigables para el medio ambiente. En este proyecto se ha realizado la construcción de un emulador de baterías ya que de esta manera se almacena en las fuentes de energías renovables, luego, mediante el diseño y construcción de un inversor trifásico se realiza la transformación de energía de un voltajecontinuo a uno sinusoidal para la alimentación de cargas a $60 \mathrm{~Hz}$. Finalmente, el uso de una trasferencia automática con

monitoreo SCADA es incorporado al sistema. Este permite la selección de la fuente primaria, teniendo como referencia la energía proveniente de la red, en caso de una falla o por voluntad del usuario, la energía eléctrica proporcionada por el inversor entraría en funcionamiento por medio de un sistema SCADA, además de visualizar los parámetros. 


\section{Introduction}

Para la utilización de energías renovables se acostumbra a usar almacenamientos de energía en diferentes formas como las baterías. Estos sistemas normalmente son costosos, por lo que se puede optar por otra alternativa que es la emulación de un banco de baterías con electrónica básica, capaz de soportar cambios de carga como lo haría un banco de baterías real a un mayor tiempo de vida. Un emulador está conformado por elementos como: Puente rectificador trifásico, barras de cobre y un condensador electrolítico. Mediante un emulador de baterías tenemos una fuente de corriente continua inagotable [1]; El siguiente paso para la utilización de energías renovables es la construcción de un inversor trifásico el cual convierte la energía de corriente continua (CC) enviada por el emulador, en corriente alterna (CA) hacia las cargas [2].

Un inversor, gracias a la utilización de dispositivos de electrónica de potencia en diferentes etapas, consigue transformar la energía de las baterías en una señal trifásica con niveles de potencia utilizables en microrredes. Estas etapas son: una tarjeta de control o (DSP) [3] [4] que se encargara de realizar el control y generación de pulsos PWM [5] y enviar los datos unidireccionalmente hasta la siguiente etapa que son las tarjetas de accionamientos. Las tarjetas de accionamiento se encuentran conformadas por un opto acoplador que finalmente se encargara de controlar de manera aislada y segura las compuertas en los módulos de transistores IGBT [6].

El PWM tiene una amplia gama de aplicaciones, incluyendo la conmutación de patrones para la operación de inversores. Los inversores se utilizan para variación de velocidad y otras aplicaciones como: cambiadores de frecuencia estática, fuentes de alimentación de reservas, fuentes de alimentación interrumpibles [3].

Finalmente, el componente adicional en inversores es el banco de transformadores, para aislar y brindar protección a los componentes electrónicos [7] de posibles perturbaciones producidas en la red.

El sistema de transferencia de energía eléctrica consiste en la selección de dos fuentes 
alimentación que sirve de suministro a un lugar en específico ya sea de manera manual o automática [8], en su modo manual el usuario tiene plena potestad de activar o desactivar la energía que este requiera [8] y en su modo automático aplica las características de STAND BY que significa que en caso de una falla en la red, el sistema realiza sus maniobras de acoplamiento y la integración de laenergía auxiliar. El sistema de transferencia de esta energía se divide en dos partes, la etapa de control y fuerza. En la parte de control se tiene como elemento central un controlador lógico programable [9], compartiendo funciones como la adquisición de datos para el monitoreo mediante la comunicación punto a punto con un analizador de red en tiempo real. Como elemento adicional, se tiene los detectores de fase [10] utilizados para detectar el sincronismo del inversor con la red domiciliaria. Un grupo de contactores se encargan de realizar de manera controlada la conexión de la fuente auxiliar o la red principal hacia la carga.

En este proyecto se enfoca en el diseño, construcción y puesta en funcionamiento, para la conexión a la red, se incluye un banco de transformadores de 3KVA, un inversor trifásico de 2KVA a 220V donde fue diseñada su etapa de control y accionamiento para operar de forma aislada mediante la alimentación por un emulador de banco de baterías y finalmente llevado a una transferencia con un diseño SCADA mediante adquisición de datos al software movicom este nos ayuda al control y monitoreo de la transferencia en tiempo real, además de observar las magnitudes eléctricas principales desde las barras de salida con la ayuda de transformadores de corriente. Las protecciones utilizadas en este proyecto son los fusibles que son dispositivos de protección echo con un alambre que se funde al momento de percibir una corriente que sobrepasa su capacidad, estos elementos de protección son ubicados antes de las barras de salida de la transferencia y así evitar daños en los dispositivos ubicados aguas arriba.

\section{DISEÑO}

El diseño tanto del módulo inversor trifásico, como del sistema de transferencia automático fue realizado en el software de AutoCAD, con la finalidad de bosquejar y elegir un modelo compacto, que abarque el espacio asignado en el tablero eléctrico, para cada uno de los sistemas, para ello se realizaron previas mediciones de los elementos físicos a implementarse. además de las revisiones de las hojas de datos emitidas por los proveedores para tener una mayor precisión.

Cabe resaltar que el módulo inversor trifásico y el sistema de transferencia fueron realizados individualmente, tanto en formato 2D como en 3D para darle un mayor realismo.

En primer lugar, se diseñó el módulo inversor trifásico ubicando los componentes de manera que su diseño presente una buena estética y ocupe las dimensiones apropiadas para las respectivas conexiones entre dispositivos y placas utilizadas. Tal como se corrobora en la siguiente figura. 


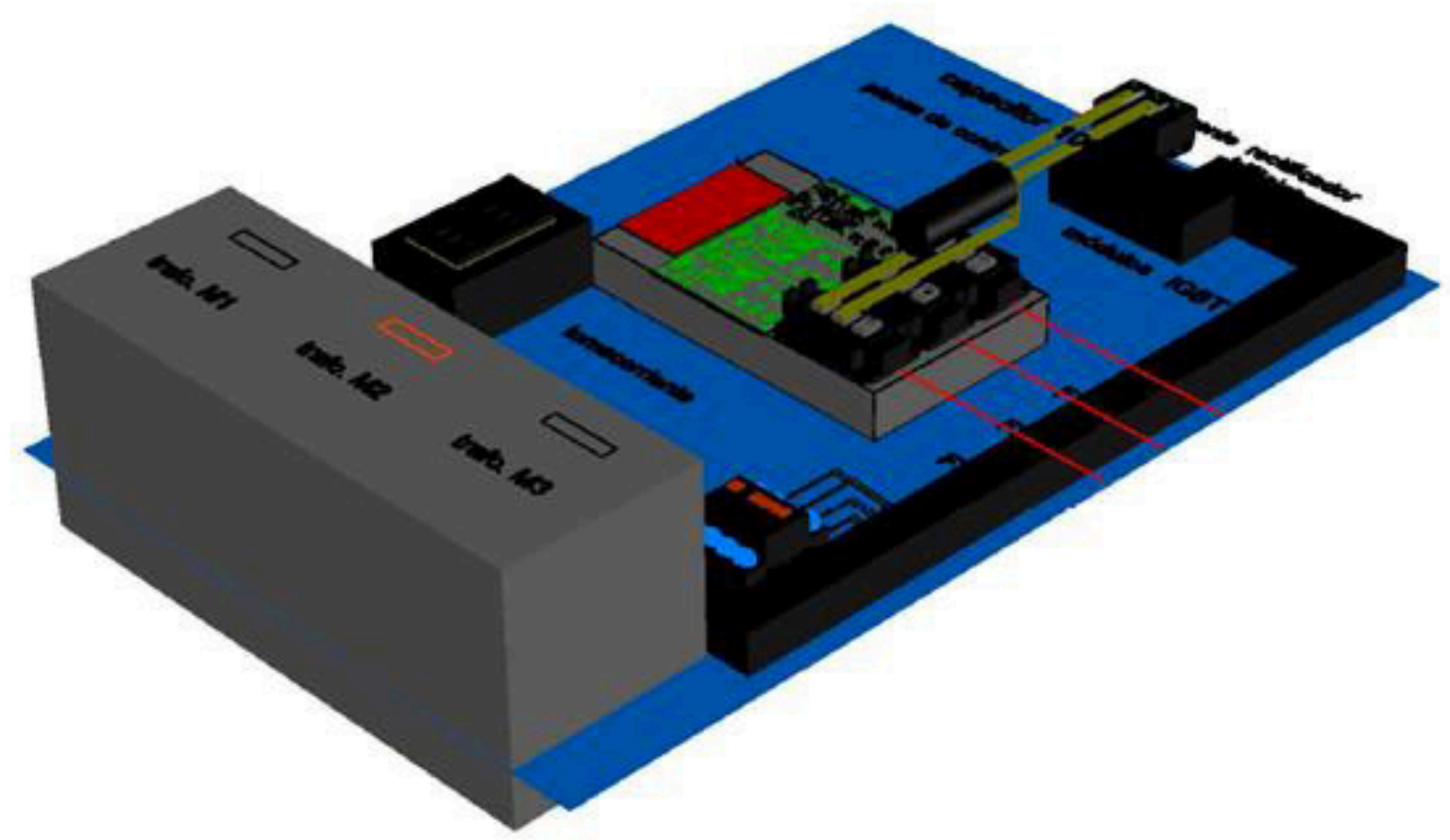

Figura 1: Diseño de componentes de modulo inversor trifásico.

En la parte inferior del modelo mostrado en la figura 1 se puede observar la disposición del banco trifásico de transformadores de aislamiento conformado por tres transformadores monofásicos de 1KVA cada uno, mismos que serán energizados por medio del uso de un breaker trifásico que se encentra en la parte superior izquierda de los mismos, para luego alimentar al puente rectificador trifásico que se encuentra en la parte superior y por consiguiente las barras del bus DC y el capacitor electrolítico, componentes que conforman el emulador del banco de baterías propuesto. En la parte central de la figura se muestra la conformación del módulo inversor el cual está compuesto un DSP, una placa de interfaz, tres placas de accionamiento y tres módulos IGBT, todos ellos se encuentran montados sobre la estructura de un disipador de calor formando un modelo compacto y firme. Además, se incluyó un tomacorriente que será utilizado para alimentar una fuente que energizara tanto las tarjetas DSP, de control como tarjetas de accionamiento de los IGBT.

Previo al montaje es necesario aclarar que se diseñó tanto las tarjetas de accionamiento como la tarjeta de interfaz en los softwares PCB Wizard y Kicad respectivamente.

A continuación, se procedió a realizar el diseño del tablero de transferencia automático definiendo la ubicación de los elementos según el funcionamiento que desempeña, este diseño se realizó en el mismo programa Autocad en el formato 3D, ya que en este se incorpora las medidas reales de cada uno de los dispositivos y de esta manera distribuir los elementos adecuadamente, en la siguiente figura se muestra la ubicación de cada elemento de la transferencia. 


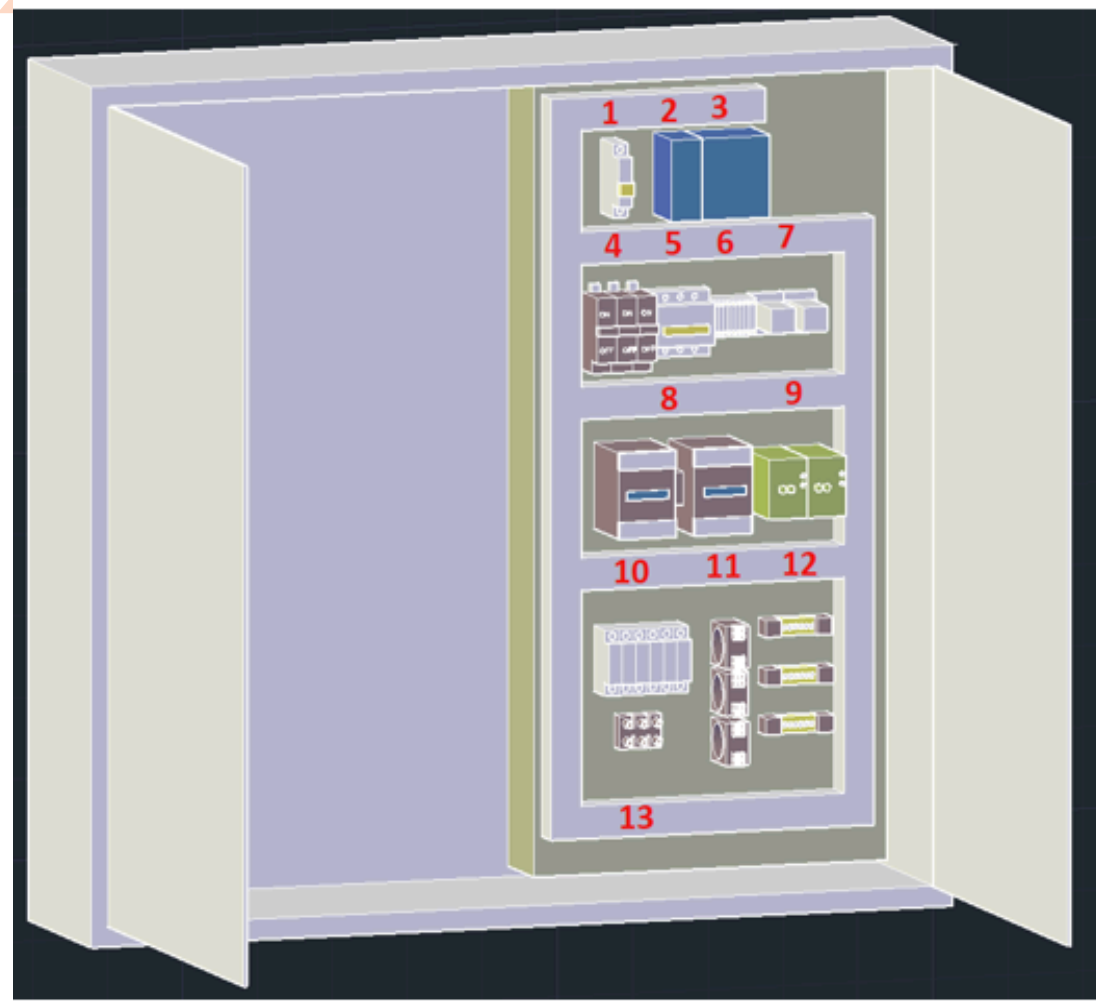

1.- Breaker monofásico

2.-Módulo de comunicación RS 485

3.- PLC Siemens S7 1200

4.- Breakers monofásicos

5.- Breaker trifásico

6.- Borneras de control

7.- Relés

8.- Contactores trifásicos

9.- Monitores de tensión

10.- Fusibles

11. $\mathrm{TCs}$

12.- Borneras de salida

13.- Borneras de unión

Figura 2: Diseño de sistema de transferencia automática

Esta ubicación se realizó en base del diagrama esquemático de control y fuerza, permitiendo de esta manera desarrollar un buen funcionamiento y distribución de los elementos, dichos elementos de todo el módulo se encuentran sobredimensionados, esto se implementó para garantizar mayor seguridad en un caso trabajar al máximo de carga establecido para este proyecto y que su vida útil sea mayor.

En su parte superior está el breaker que funciona como protección y alimentación al plc, el cual es de marca Siemens modelo S7 1200 que se alimenta a un voltaje fase - neutro (120 volt.), además de un módulo de comunicación RS 485 que ayuda al sistema de adquisición de datos.

En la siguiente parte se tiene elementos de protección y control, estos son los disyuntores monofásicos que son tres en total uno para cada línea, se puso de esta manera para poder verificar el funcionamiento y accionamiento de este módulo, también un breaker trifásico que ayuda en la protección de la fuente auxiliar proveniente del inversor, ya que en caso de una variación de corriente excesiva estos se abran y no ocasiones daños a los componentes que conforma el inversor, además se tiene borneras que permiten la conexión y distribución de las entradas y salidas del PLC y por último se tiene relés los cuales ayudan a la activación de los contactores y luces indicadoras, esto se realiza a través de las indicaciones que da el PLC.

Como tercer bloque se encuentran los contactores, quienes permiten el paso de la energía eléctrica ya sea de la red principal suministrada por cualquier empresa eléctrica o por una red auxiliar que en este caso es suministrada por un inversor trifásico conectado a un emulador de banco de baterías. 
En el último bloque se tiene elementos de protección que son fusibles, quienes son lo encargados de proteger todos los dispositivos aguas arriba en caso de un cortocircuito en las cargas, y por últimos tenemos las barras de salida a las cuales se conecta cualquier tipo de carga.

\section{CONSTRUCCIÓN Y PRUEBAS DE FUNCIONAMIENTO}

Para el proceso de construcción, el trabajo fue realizado por etapas, iniciando con el montaje de los dispositivos en las tres placas de accionamiento de IGBT, iniciando por la soldadura del optoacoplador que es el dispositivo principal y más sensible en dicha placa y luego la soldadura de los dispositivos adicionales tales como conectores, resistencias luces indicadoras, capacitores y bases de pines para el convertidor DC-DC y la compactación de estas por medio de un soporte plástico.

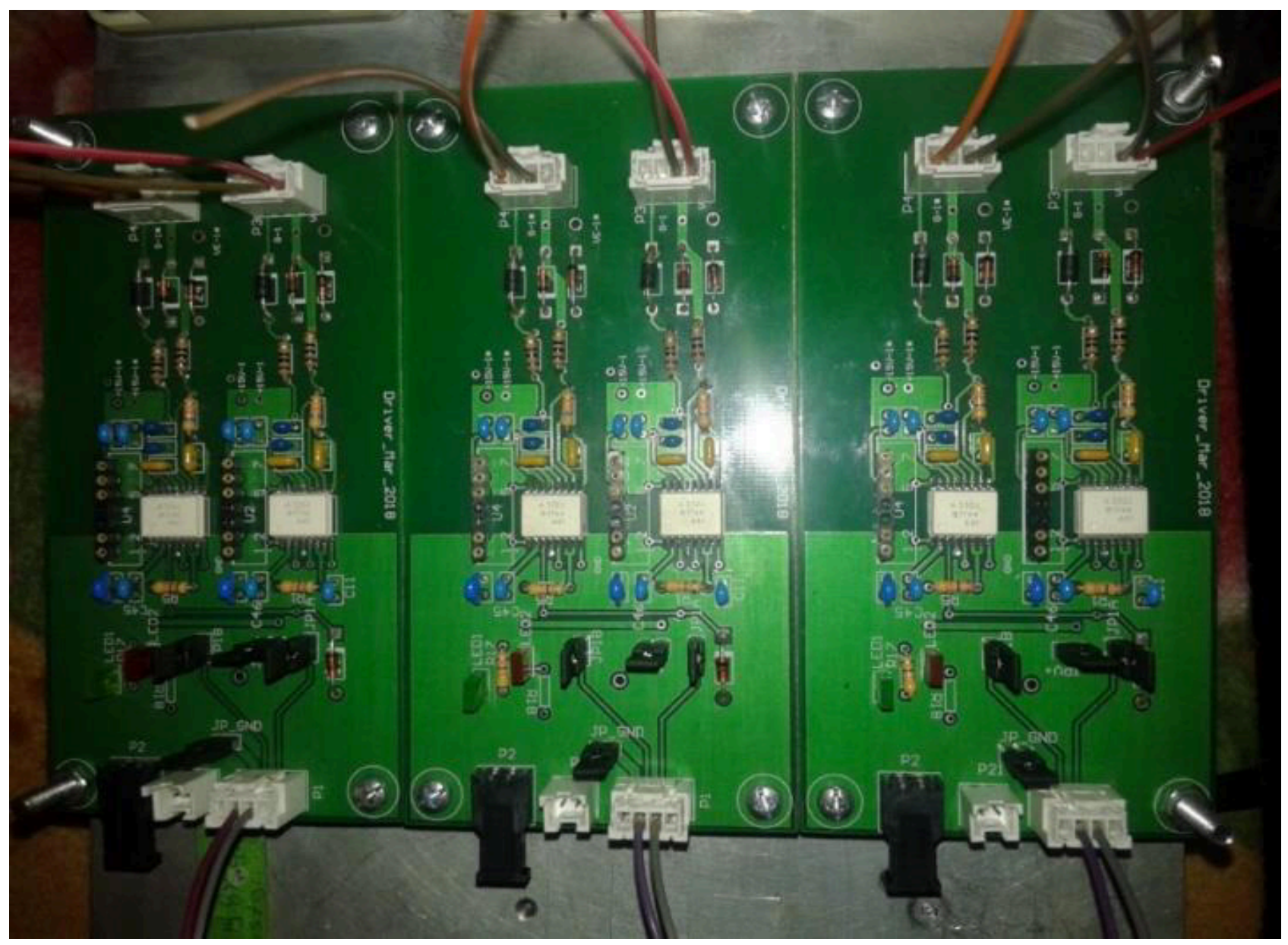

Figura 3: Placas de accionamiento IGBT

Luego se realizó el montaje de los dispositivos correspondientes a la tarjeta de interfaz acoplada con la tarjeta DSP; teniendo el sistema montado en el gabinete, se procede a realizar las pruebas de funcionamiento en vacío. Finalmente, en base al modelo diseñado en AutoCAD se procede a realizar el montaje de los dispositivos de potencia iniciando por elmontaje del módulo rectificador trifásico en su respectivo disipador, seguidamente la medición y curvatura correspondiente de las barras de cobre y perforaciones de acuerdo con los espacios necesarios para el ajuste de los tornillos tanto del capacitor electrolítico como de los módulos IGBT, a todo ello se le anexo la parte de control por medio de un 
disipador de mayor magnitud obteniendo como resultado un prototipo compacto tal como se puede observar en la siguiente figura.

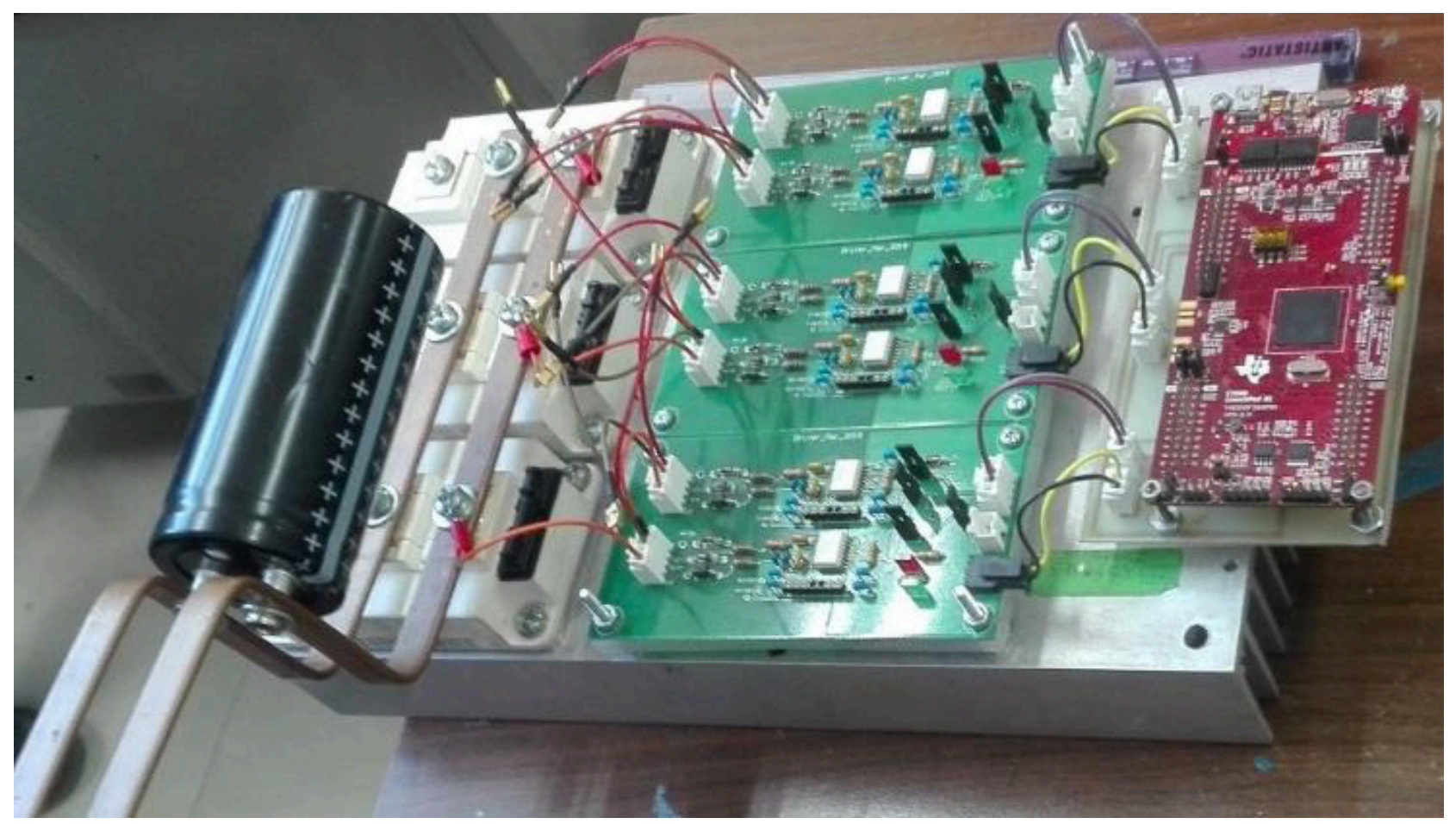

Figura 4: Conformación de Inversor trifásico

En la etapa de contrucción se realizo el montaje de los elementos que conforma el sistema de transferencia automática, esta implementación se realizo con base en el diseño anteriormente explicado, esta construción de divide en parte interna y externa.

Una vez teniendo el montaje de los elmentos, en la parte interna se realizan las conexiones desde el PLC hasta los diferentes elementos.

Con la etapa de control en funcionamiento, se prosigue a realizar las conexiones del circuito de fuerza. La parte de control se prosigue con las conexiones de fuerza, lo cual implica las alimenciones de los monitores de tensión que el primer punto que es la presencia de las energía tanto la principal como la auxiliar, continuando por los contactores que al activarse dan paso a de energía para allimentar a las barras de salida. 


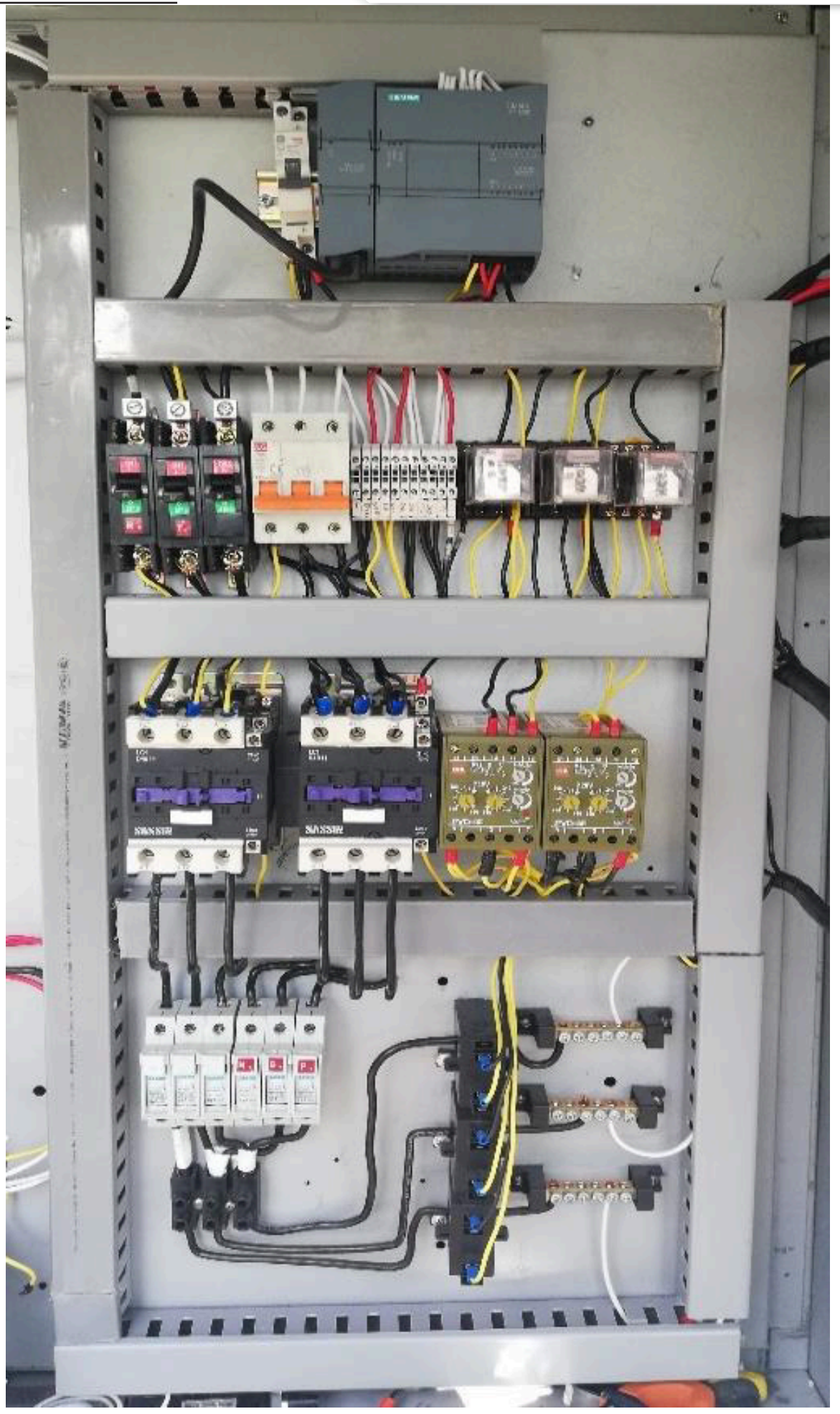

Figura 5: Elementos internos de TTA

En la parte externa del módulo se encuentran ubicados los elementos de medida como el analizador de redes, donde se obtienen distintos parámetros de medida, mediante las señales provenientes de los transformadores de corrientes ubicados en cada línea. En esta parte del gabinete también se encuentran el módulo de pulsadores cumpliendo funciones de arranque, paro y suministro de energía eléctrica proveniente de la red principal o de la fuente auxiliar según las condiciones de operación. Estas funciones son mostradas por los indicadores de señalización 


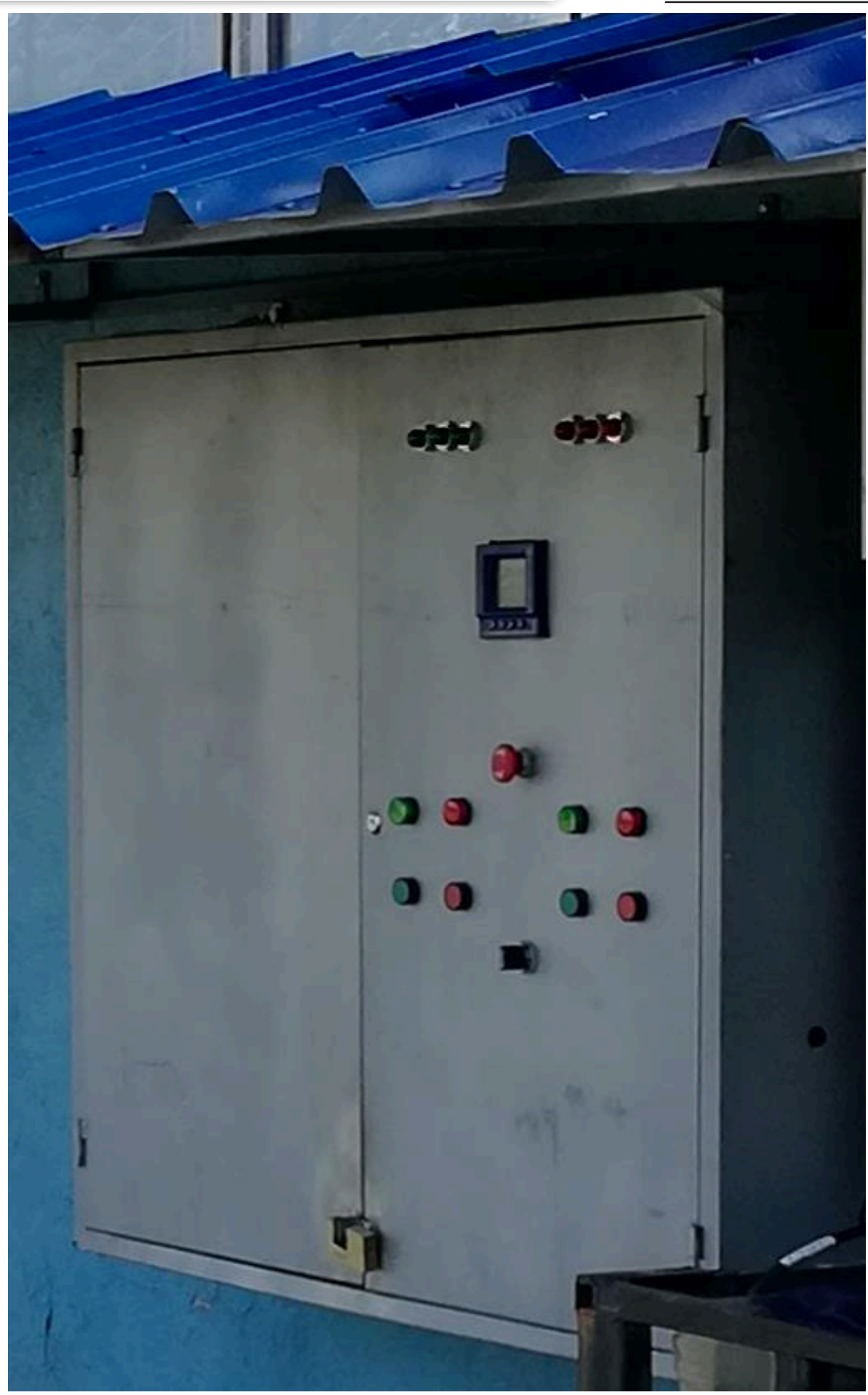

Figura 6: Elementos extrenos de TTA

El diseño SCDA se realizó con el software movicon, se optó por este, ya que es muy versátil y de fácil uso, con este SCADA se puede controlar y monitorear el sistema de forma local y remota

Las pruebas de funcionamiento se realizaron en varias etapas. La primera prueba consistió en verificar las ondas de salida, sin alimentación en el bus DC. La siguiente actividad se aplicó voltaje variable a la barra DC, observando las señales en los colectores de los módulos IGBT's, la corriente en el bus DC tal como se muestra en la figura7. 


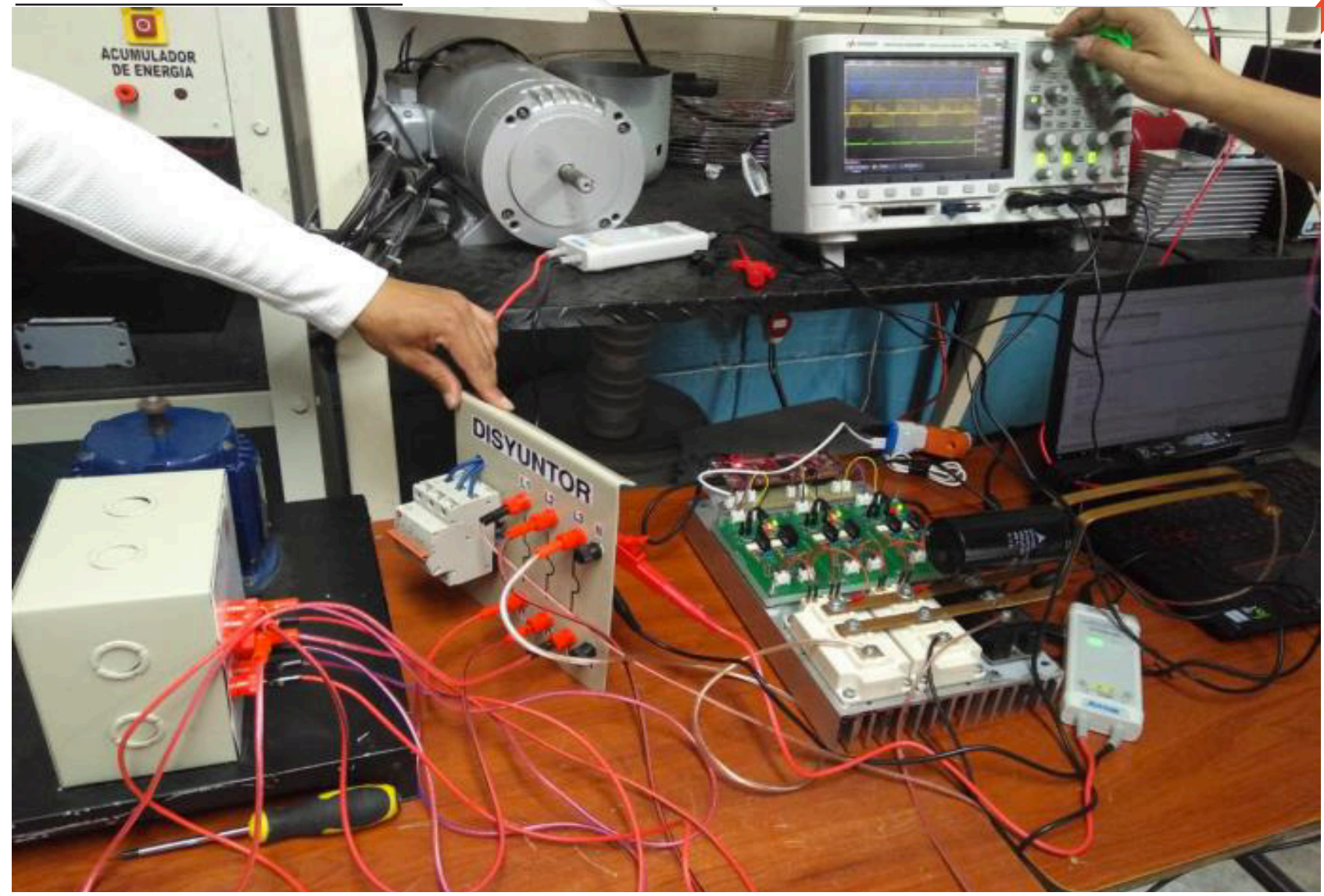

Figura 7: Pruebas de funcionamiento de inversor

Finalmente, se conecta una carga de 15W, ejecutando las pruebas anteriores, para lograr condiciones nominales de operación, se operó el bus DC a 120V.

DSO-X 2024A, M/56272661: Sat Jan 26 00:26:192019

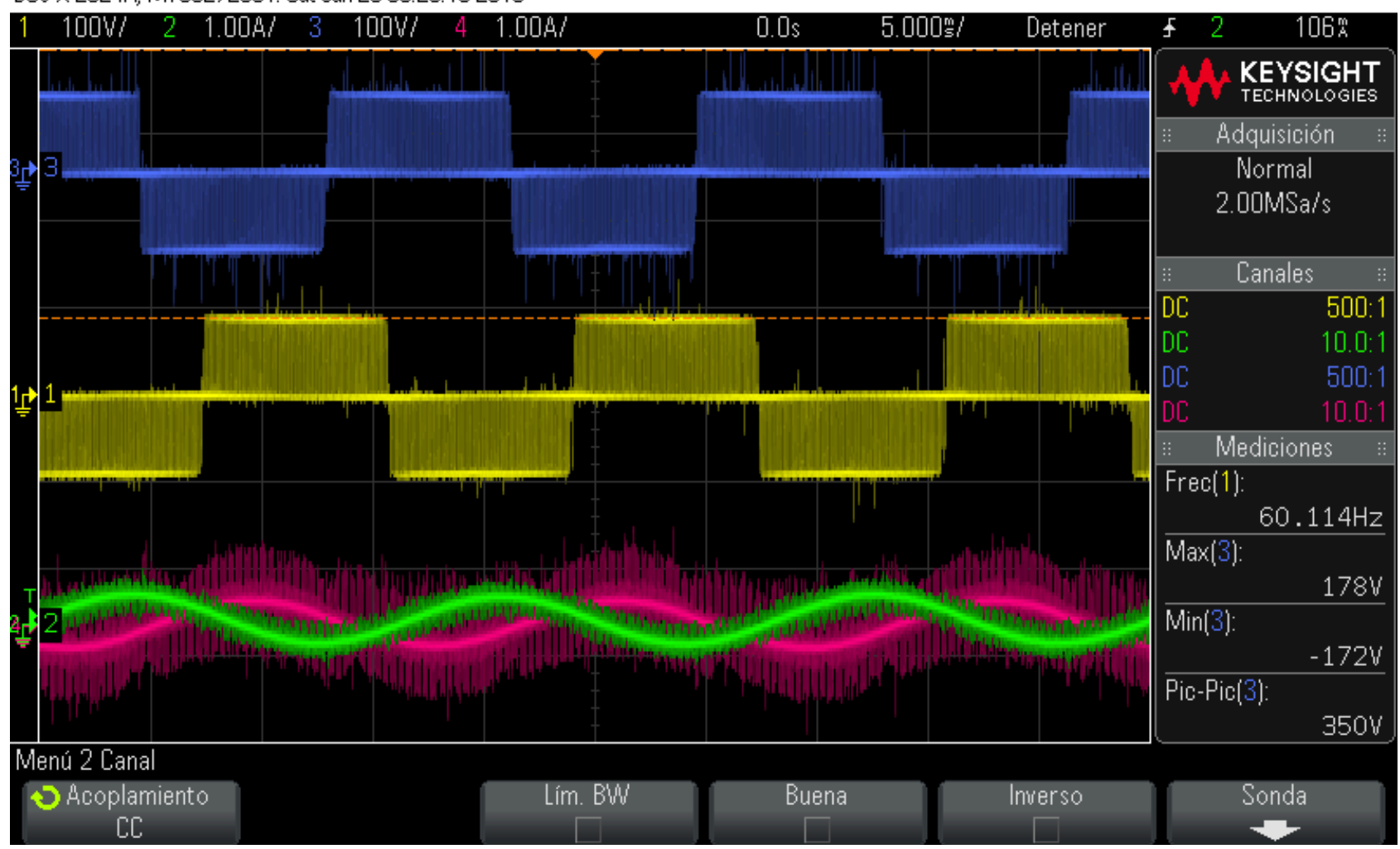

Figura 8: Resultado de voltajes y corrientes con carga en inversor 


\section{Ch 2 escala 100V/, Ch 3 escala 1.00A/, Pos 2.86250 A, nivel49.9800V, escala principal} $5.000 \mathrm{~ms}$.

Para un abanico amplio de funcionamiento se realizaron varias pruebas de verificación, con diferentes tipos de cargas y a diferentes potencias.

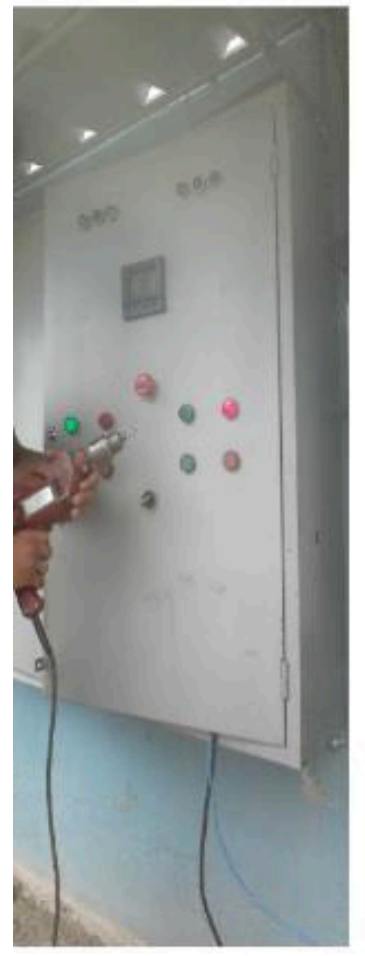

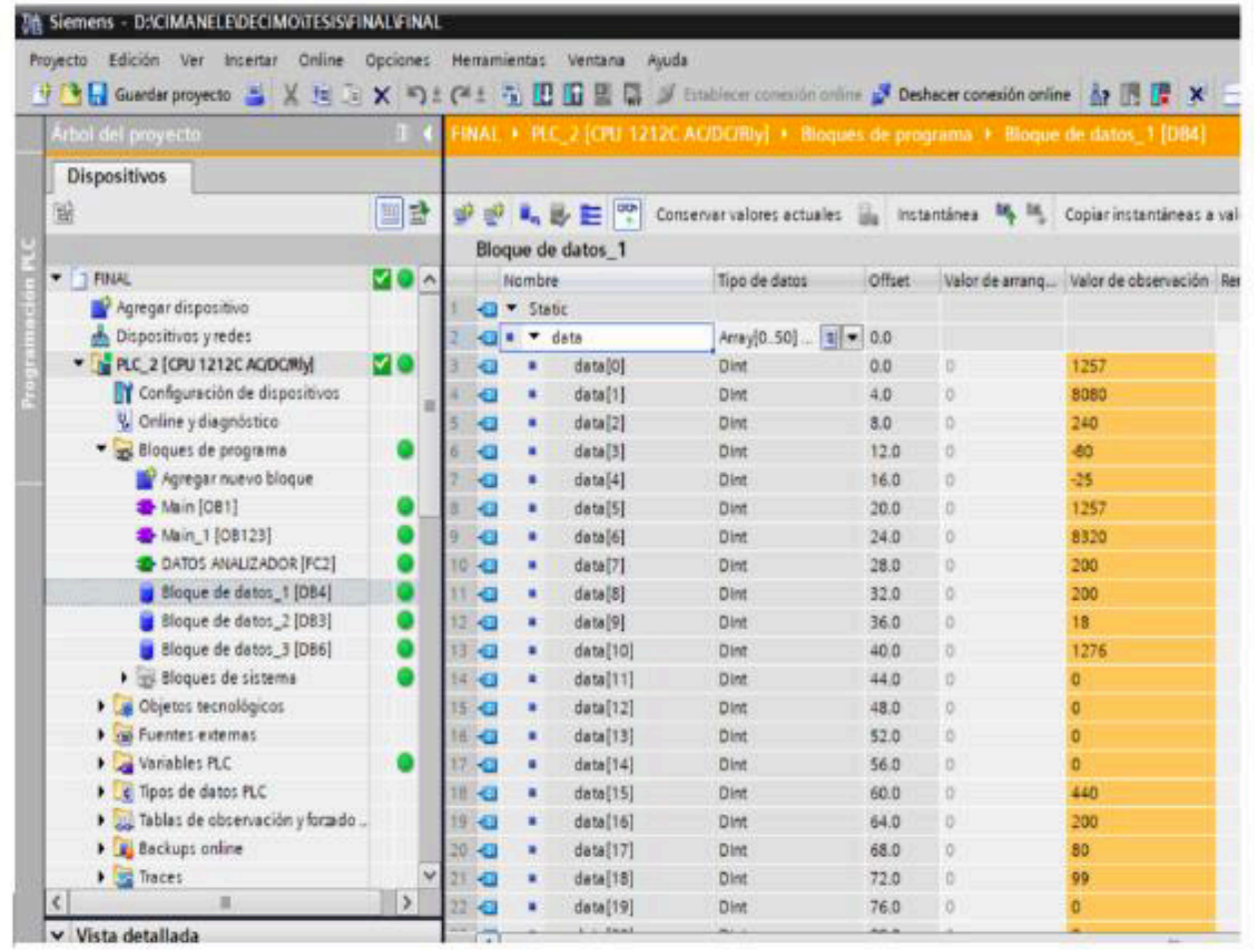

Figura 9: Parámetros de medición en taladro

Otra de las pruebas fue la comunicación SCADA, la cual se encarga del accionamiento de forma remota, esto implica tener una conexión punto a punto a través del PLC y el computador, teniendo así un buen desempeño del sistema. 


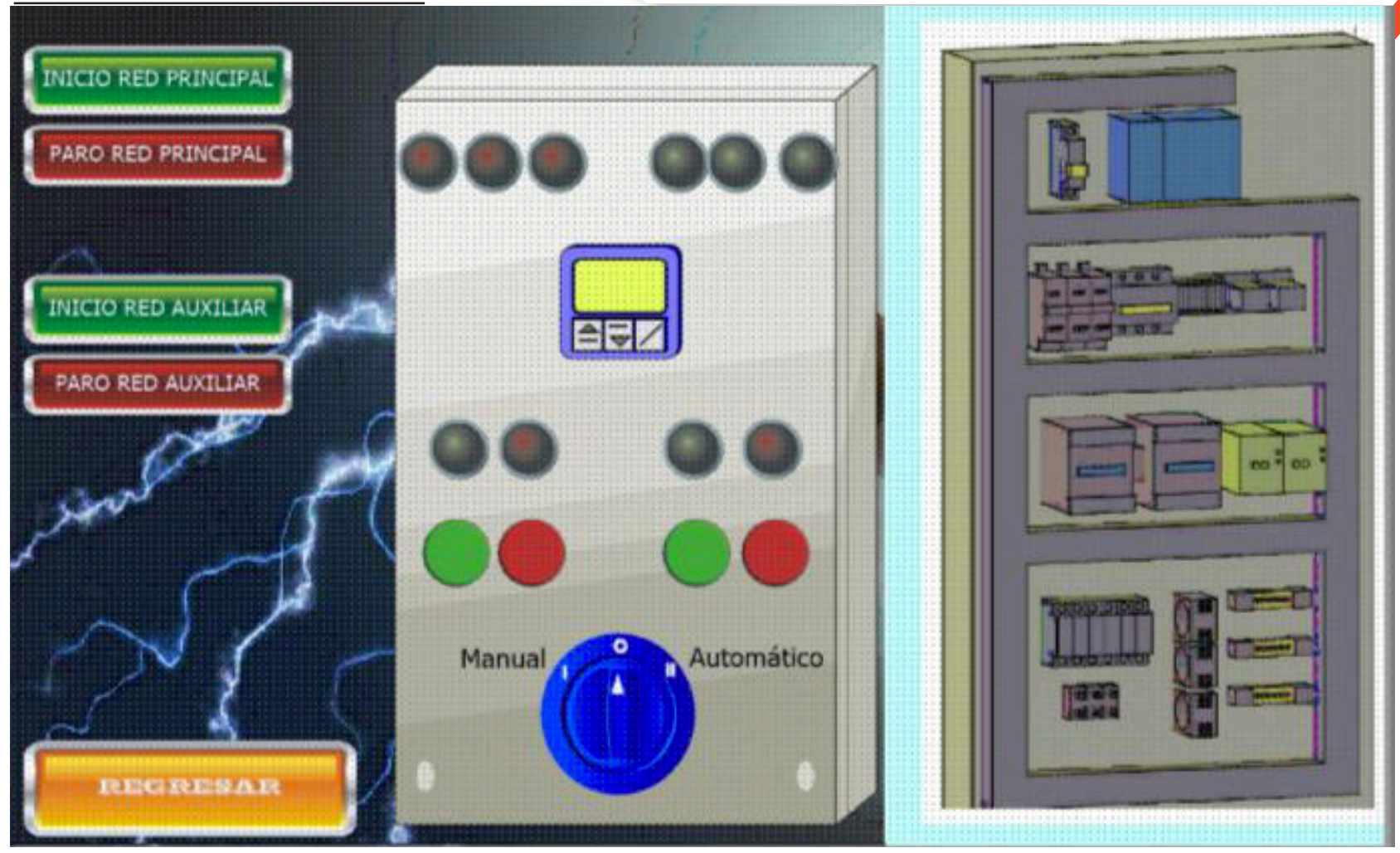

Figura 10: Pantalla SCADA para comunicación remota

\section{CONCLUSIONES Y TRABAJOS FUTUROS}

Se confirmaron los diferentes bloques para el diseño y construcción de un inversor trifásico con sistema SCADA.

Al ser un sistema con funcionamiento aislado a la red, fue necesaria la utilización de un tablero de transferencia automática.

El diseño del sistema completo fue proyectado de forma didáctica y genérica, motivo por el cual, trabajos futuros de relevancia como la implementación de un control de lazo cerrado e incluso inyección de potencia a la red.

El aislamiento magnético del sistema por medio de transformadores es de gran importancia para la protección integral de componentes del proyecto.

Un emulador de banco de baterías resuelve la problemática de la vida útil y costos de un banco de baterías por lo que es la mejor opción para la implementación de prototipos.

Por medio del sistema SCADA es posible monitorear que el sistema implementado se encuentre en condiciones normales de funcionamiento.

Se pretende con el módulo obtenido, el incursionar con prácticas de laboratorio para asignaturas referentes a laelectrónica de potencia y el uso de energías no convencionales. 


\section{BIBLIOGRAFÍA}

1. Vernavà, A., Gibbons, R. N., \& Novello, A. Electronica de Potencia. Inversion CC/CA monofasica, (pág. 27) (2006).

2. Fowler, R. ELECTRICIDAD Principios y Aplicaciones. Barcelona: Reverté, S.A. (1994).

3. Torres, C., Murillo, D., \& Restrepo, C. Pereira- Colombia Patente n ISSN: 0122-1701. (28 de febrero de 2008).

4. Recuperado el Lunes de Enero de 2017, de Guia Para Diseño de Redes para Distribucion: http://ftp.eeq.com.ec/upload/informacionPublica/2014/NORMAS\%20PARA\%20 SISTEMAS\%20DE\%20DISTRIBUCION\%20PARTE\%20A.pdf

5. Texas Instruments. LAUNCHXL-F28379D Overview. LAUNCHXL-F28379D Overview (junio de 2018)

6. NATIONAL INSTRUMENTS. What is a Pulse Width Modulation (PWM) Signal and What is it Used for? NATIONAL INSTRUMENTS, 2. (2018).

7. Hermosa, A. Electronica Aplicada (CF Instalaciones de Electronica y Telecomunicaciones). Barcelona: MARCOMBO S.A. (2012).

8. Daura, F. Los Transformadores de Aislamiento y las EMls. CEMDAL, 6. btenido de electricasas: http://www.electricasas.com/instalacioneselectricas-electricidad-2/ gruposelectrogenos/tablero-de-transferenciaautomatica-tta/ (17 de 05 de 2018)

9. Soria Tello, S. Sistemas Automáticos Industriales de Eventos Discretos. Mexico: Alfaomega Grupo Editor S.A, de C.V. (2013).

10. Promelsa. COEL. Obtenido de http://www.promelsa.com.pe/pdf/1008804.pdf (01 de 09 de 2006). 